\title{
Announcing the Journal of Hospital Medicine Editorial Fellowship
}

\author{
Charlie M Wray, DO, MS¹,2; Andrew Olson, MD³; Samir S Shah, MD, MSCEE 4 ; Andrew D Auerbach, MD, MPH
}

'Department of Medicine, University of California, San Francisco, San Francisco, California; ${ }^{2}$ Division of Hospital Medicine, San Francisco Veterans Affairs Medical Center, San Francisco, California; ${ }^{3}$ Division of Internal Medicine, University of Minnesota, Minneapolis, Minnesota; ${ }^{4}$ Division of Hospital Medicine, Cincinnati Children's Hospital Medical Center, Cincinnati, Ohio; ${ }^{5}$ nniversity of Cincinnati College of Medicine, Cincinnati, Ohio; ${ }^{6}$ Division of Hospital Medicine, University of California, San Francisco, San Francisco, California.

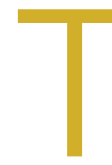

he peer review and editorial processes are integral activities in academic medicine that provide ethical, independent, and unbiased critical assessment of submitted manuscripts to academic journals. Recognizing that few trainees or junior faculty are formally exposed to these processes, ${ }^{1}$ the Journal of Hospital Medicine aims to fill this opportunity gap through the launch of a one-year Editorial Fellowship.

The Fellowship is open to chief residents, hospital medicine fellows, and junior faculty (eg, Assistant Professor or Clinical Instructor). Starting in July of each year, a group of four to six applicants are paired with editorial mentors who are current JHM Deputy or Associate Editors. Structured as a distance-learning program, this program aims to allow Fellows the ability to continue in their full time professional roles while also allowing the opportunity to engage with national leaders in hospital medicine. Regular communication and interactions take place through both synchronous and asynchronous means. Fellows' responsibilities during the 12-month experience include: completion of six guided peer reviews, preparation of one or two editorials, participation in monthly editorial meetings, and quarterly educational videoconferences. Interested Fellows may also have an opportunity to co-lead the journal's online journal club, \#JHMChat. ${ }^{2}$ Fellows are expected to attend the editorial staff meeting at the annual Society of Hospital Medicine Conference.

*Corresponding Author: Charlie M. Wray, DO, MS; E-mail: Charlie.Wray@ucsf. edu; Telephone: 415-595-9662; Twitter: @WrayCharles

The application may be found in the online version of this article.

Received: November 18, 2018; Revised: November 26, 2018;

Accepted: December 2, 2018

(๑) 2019 Society of Hospital Medicine DOI 10.12788/jhm.3132
With this program, JHM aims to accomplish several tasks. First, we hope to offer a unique educational experience that allows for further growth, development, inspiration, and experience in academic medicine-specifically around the manuscript review and editorial processes. Second, recognizing that a journal's quality is frequently a product of its reviewers, JHM hopes to build a cadre of well-trained and experienced reviewers and, hopefully, future members of the JHM editorial leadership team. Third, the program hopes to act as a networking experience, allowing editorial Fellows to learn from, collaborate with, and become academic leaders in the field. Finally, we hope to provide an opportunity for Fellows to be academically productive in their composition of editorial content-an output that will help catalyze their professional development.

We believe that in working closely with the JHM editorial staff, this program will help develop the next generation of leaders in academic hospital medicine. We strongly encourage applications from physicians who have been historically under-represented in leadership in academic medicine. Further details and the application can be found in the appendix and on the JHM website (www.journalofhospitalmedicine.com). It will be announced annually through the @JHospMedicine twitter handle.

Disclosures: The authors have nothing to disclose.

\section{References}

1. Lovejoy $\mathrm{TI}$, Revenson TA, France CR. Reviewing manuscripts for peer-review journals: a primer for novice and seasoned reviewers. Ann Behav Med Publ Soc Behav Med. 2011;42(1):1-13. doi:10.1007/s12160-011-9269-x

2. Wray CM, Arora VM, Auerbach AD. The Adoption of an Online Journal Club to Improve Research Dissemination and Social Media Engagement Among Hospitalists. J Hosp Med. 2018;13(11). doi:10.12788/jhm.2987 